\title{
REDUCED BOUNDARIES AND CONVEXITY
}

\author{
DAVID G. CARABALLO
}

(Communicated by Tatiano Toro)

\begin{abstract}
We establish strong, new connections between convex sets and geometric measure theory. We use geometric measure theory to improve several standard theorems from the theory of convex sets, which have found wide application in fields such as functional analysis, economics, optimization, and control theory. For example, we prove that a closed subset $K$ of $\mathbb{R}^{n}$ with nonempty interior is convex if and only if it has locally finite perimeter in $\mathbb{R}^{n}$ and has a supporting hyperplane through each point of its reduced boundary. This refines the standard result that such a set $K$ is convex if and only if it has a supporting hyperplane through each point of its topological boundary, which may be much larger than the reduced boundary. Thus, the reduced boundary from geometric measure theory contains all the convexity information for such a set $K$. We similarly refine a standard separation theorem, as well as a representation theorem for convex sets. We then extend all of our results to other notions of boundary from the literature and deduce the corresponding classical results from convex analysis as special cases.
\end{abstract}

\section{INTRODUCTION}

A closed subset $K$ of $\mathbb{R}^{n}$ with non-empty interior is convex if and only if it has a supporting hyperplane at each point of its topological boundary $\partial K$ (see, for example, [12] Theorem 5.4, [14] Theorem 1.3.3 and Corollary 1.3.5, [15] Theorem 2.4.12). R. Webster ([15], p. 71) calls this result "arguably the single most important property of convex sets."

The result is surprising since it shows that the topological boundary, which seems to have little to do with whether line segments bounded by points in $K$ must entirely be in $K$, essentially contains all the information about the convexity of a closed set with non-empty interior.

This extremely important theorem is intimately connected to fundamental theorems concerning, for instance, separation, representation, and nearest point projection, with important applications in fields such as functional analysis, economics, optimization, and control theory (see, for example, [14, 16]).

In this paper, we improve this result by showing that it is not the topological boundary, but rather the reduced boundary from geometric measure theory, that contains all the convexity information about a closed set with non-empty interior. Specifically, we have the following (also, see Theorem 4):

Received by the editors October 26, 2010 and, in revised form, February 11, 2011.

2010 Mathematics Subject Classification. Primary 52A20, 52A30, 28 A75.

Key words and phrases. Convex, reduced boundary, measure-theoretic boundary, topological boundary, support, separation, nearest point, supporting hyperplane, supporting half-space, density. 
Theorem 1. Suppose $K \subset \mathbb{R}^{n}$ is closed, with non-empty interior $K^{\circ}$. Then $K$ is convex if and only if $K$ has locally finite perimeter in $\mathbb{R}^{n}$ and $K$ has a supporting hyperplane at each point $p$ of its reduced boundary $\partial_{\text {red }} K$.

In this way, we also significantly extend the main results from the paper [3].

It is surprising that we can deduce the convexity of $K$ without even considering supporting hyperplanes through points in the potentially very large set $\partial K \backslash \partial_{\text {red }} K$. Indeed, inf $\left\{\mathcal{H}^{n-1}\left(\partial_{\text {red }} K\right) / \mathcal{H}^{n-1}(\partial K): K \subset \mathbb{R}^{n}\right\}=0$ (see, for example, [10] 1.10).

We use reduced boundaries to similarly refine other standard results from convex analysis concerning separation and representation (see Theorems 2, 3, and 4).

Finally, in Section 4 we strengthen our results by considering more general notions of boundary. We show quite generally that each of our previous theorems holds with $\partial_{\text {red }} K$ replaced by any set $A \subset \mathbb{R}^{n}$ satisfying

$$
\partial_{\text {red }} K \subset_{n-1} A \subset \partial K
$$

(see Theorems [6 and 8). This includes, as special cases, most commonly used notions of boundary from the literature, such as the measure-theoretic boundary $\partial^{M} K$, the boundary $\partial_{F} K$ in the sense of Federer ([9] 4.5.6), and the set $K^{1 / 2}$ of points where $K$ has $n$-dimensional density equal to $1 / 2$. Of course, $A=\partial K$ itself satisfies (11), so that we immediately recover, as corollaries to our theorems, major results from the convex analysis literature concerning separation and representation (see Corollaries 7 and 9).

These new connections between convex sets and geometric measure theory are quite useful. Recently, we have made use of these connections to establish new local criteria for convexity (see [4]).

\section{Notation AND REDUCED BOUNDARIES}

Throughout this paper, we work in $\mathbb{R}^{n}$ with $n \geq 2$. If $A \subset \mathbb{R}^{n}, \bar{A}$ and $A^{\circ}$ denote the topological closure and topological interior of $A$ in $\mathbb{R}^{n}$. The interior of $A$ relative to the smallest affine subspace, or flat, containing $A$ is denoted relint $A$. When $A \subset \mathbb{R}^{n}$, we let $\partial A=\bar{A} \cap \overline{\mathbb{R}^{n} \backslash A}$ denote the topological boundary of $A$. We note that $x \in \partial A$ if and only if for each $r>0$ we have $U(x, r) \cap A \neq \emptyset$ and $U(x, r) \cap\left(\mathbb{R}^{n} \backslash A\right) \neq \emptyset$.

If $A, B \subset \mathbb{R}^{n}$ and $0<m \leq n$, we write $A \subset_{m} B$ (i.e., " $A$ is $\mathcal{H}^{m}$ almost contained in $B^{\prime \prime}$ ) when $\mathcal{H}^{m}(A \backslash B)=0$. We will measure volume and surface area in $\mathbb{R}^{n}$ with $n$-dimensional Lebesgue measure $\mathcal{L}^{n}$ and $(n-1)$-dimensional Hausdorff measure $\mathcal{H}^{n-1}$, respectively. We let $U(p, r)$ and $B(p, r)=\overline{U(p, r)}$ denote, respectively, the open and closed balls in $\mathbb{R}^{n}$ with center $p$ and radius $r \in(0, \infty)$, and we set $\alpha(n)=\mathcal{L}^{n}(B(\mathbf{0}, 1))$, where $\mathbf{0}=(0,0, \ldots, 0)$ is the origin in $\mathbb{R}^{n}$.

Given a point $p \in \mathbb{R}^{n}$ and a unit vector $u \in \mathbb{R}^{n}$, we define the hyperplane $H(p, u)=\{x:(x-p) \cdot u=0\}$ and its associated open half-spaces $H_{+}(p, u)=$ $\{x:(x-p) \cdot u>0\}$ and $H_{-}(p, u)=\{x:(x-p) \cdot u<0\}$. A hyperplane $H=H(p, u)$ is a supporting hyperplane for the set $X \subset \mathbb{R}^{n}$ at the point $p \in \mathbb{R}^{n}$ provided $p \in X$, $p \in H$, and either $X \subset \overline{H_{+}(p, u)}$ or $X \subset \overline{H_{-}(p, u)}$. A closed half-space $F$ in $\mathbb{R}^{n}$ is called a supporting half-space for a set $X \subset \mathbb{R}^{n}$ if $X \subset F$ and if $F$ is bounded by a supporting hyperplane for $X$. 
When $X \subset \mathbb{R}^{n}$ is $\mathcal{L}^{n}$ measurable, $p \in \mathbb{R}^{n}$, and $R>0$, we define the $n$-dimensional density ratio of $X$ at $p$ in $B(p, R)$ as

$$
\Theta^{n}(X, p, R)=\mathcal{L}^{n}(X \cap B(p, R)) / \mathcal{L}^{n}(B(p, R)),
$$

and we set $\Theta^{n}(X, p)=\lim _{R \rightarrow 0^{+}} \Theta^{n}(X, p, R)$, provided the limit exists.

Suppose $\Omega$ is an open subset of $\mathbb{R}^{n}$. Whenever $u \in L_{\text {loc }}^{1}(\Omega)$ and $U \subset \Omega$ is open, we define the total variation of $u$ in $U$ as follows:

$$
T V(u, U)=\sup _{\substack{\phi \in C_{c}^{1}\left(U, \mathbb{R}^{n}\right) \\|\phi(x)| \leq 1 \text { for all } x \in U}}\left\{\int_{x \in U} u(x) \operatorname{div} \phi(x) d \mathcal{L}^{n} x\right\} .
$$

We can extend $T V(u, \cdot)$ to be a Borel measure on $\Omega$ by setting

$$
T V(u, E)=\inf \{T V(u, U): U \text { is an open set containing } E\}
$$

for any Borel set $E$ in $\Omega$. Then $T V(u, \cdot)$ is a Radon measure on $\Omega$ if and only if $T V(u, K)<\infty$ for all compact $K \subset \Omega$. We note that, if $u \in C^{1}(\Omega)$, then integration by parts gives $T V(u, \Omega)=\int_{\Omega}|\nabla u| d \mathcal{L}^{n}$. We let

$$
B V_{l o c}(\Omega)=\left\{f: f \in L_{l o c}^{1}(\Omega), \text { and } T V(f, K)<\infty \text { for all compact } K \subset \Omega\right\}
$$

denote the space of functions of locally bounded variation in $\Omega$.

When $A$ is an $\mathcal{L}^{n}$ measurable subset of $\mathbb{R}^{n}$, we let $P(A, \Omega)=T V\left(\chi_{A}, \Omega\right)$ be the perimeter of $A$ in $\Omega$. We say that $A$ has locally finite perimeter in $\mathbb{R}^{n}$ provided $\chi_{A} \in B V_{l o c}\left(\mathbb{R}^{n}\right)$. Suppose $A$ is an $\mathcal{L}^{n}$ measurable subset of $\mathbb{R}^{n}$ having locally finite perimeter in $\mathbb{R}^{n}$. The structure theorem for $B V_{l o c}$ functions (see, for example, 7 ] 5.1 ) ensures that there exists a Radon measure $\mu$ (which we'll denote $\mu=\left\|D \chi_{A}\right\|$ ) on $\mathbb{R}^{n}$ and a $\mu$-measurable function $\sigma: \mathbb{R}^{n} \rightarrow \mathbb{R}^{n}$ such that $|\sigma(x)|=1$ for $\mu$ almost every $x$, and

$$
\int_{A} \operatorname{div} \varphi d \mathcal{L}^{n}=-\int_{\mathbb{R}^{n}} \varphi \cdot \sigma d \mu
$$

for each $\varphi \in C_{c}^{1}\left(\mathbb{R}^{n}, \mathbb{R}^{n}\right)$. For such a set $A$, the reduced boundary of $A$ in the sense of De Giorgi (see [5], 6], 7] 5.7.1, 1] Definition 3.54), which we will denote $\partial_{\text {red }} A$, consists of all points $x \in \mathbb{R}^{n}$ for which

(1) $\left\|D \chi_{A}\right\|(U(x, r))>0$ for each $r>0$,

(2) the limit

$$
v_{A}(x)=\lim _{r \rightarrow 0} \frac{D \chi_{A}(U(x, r))}{\left\|D \chi_{A}\right\|(U(x, r))}
$$

exists, and

(3) $\left|v_{A}(x)\right|=1$.

Here, $v_{A}: \partial_{\text {red }} A \rightarrow S^{n-1}$ is called the generalized inner normal to $A$.

If $X \subset \mathbb{R}^{n}$ and $p \in \mathbb{R}^{n}$, the vector $u \in \mathbb{R}^{n}$ is called a measure-theoretic exterior unit normal to $X$ at $p$ in the sense of Federer (cf. 8, 9] 4.5.5) provided $|u|=1$, $\Theta^{n}\left(H_{+}(p, u) \cap X, p\right)=0$, and $\Theta^{n}\left(H_{-}(p, u) \backslash X, p\right)=0$. If no such $u$ exists, we define $n_{X}(p)=\mathbf{0}$; while if such a $u$ exists, it is necessarily unique (8] Theorem 3.4), and we define $n_{X}(p)=u$. Whenever $X$ is $\mathcal{L}^{n}$ measurable and $p \in \partial_{\text {red }} X, n_{X}(p)$ is a unit vector. 
Throughout this paper, we work with closed subsets $K$ of $\mathbb{R}^{n}$ having non-empty interior $K^{\circ}$. If such a set does not have locally finite perimeter in $\mathbb{R}^{n}$, then it is necessarily non-convex, and all properties which imply convexity fail to hold (see Theorem (4). Therefore, we will restrict attention to the interesting case where $K$ has locally finite perimeter in $\mathbb{R}^{n}$.

Some excellent references for reduced boundaries include [1, [7, [10, 11], and [13]. Also, see [2].

\section{Separation, CONVEXity, AND REDUCED Boundaries}

We begin by establishing a key separation result, a refinement of a well-known result about convex sets (see, for example, 14 Theorems 1.3.3 and 1.3.4) which asserts that if $K$ is a closed subset of $\mathbb{R}^{n}$ having non-empty interior and having supporting hyperplanes at each point $p$ of its topological boundary, then any $x \in$ $\mathbb{R}^{n} \backslash K$ can be separated from $K$ by a supporting hyperplane of $K$ through a point of the topological boundary.

We assume less, supposing only that $K$ has supporting hyperplanes at each reduced boundary point, and our conclusion is stronger: if $x \in \mathbb{R}^{n} \backslash K$ we are able to separate $x$ from $K$ using a hyperplane that passes through the reduced boundary. Our proof makes use of a new cone construction and the relative isoperimetric inequality.

Theorem 2 (Separation theorem for reduced boundaries). Suppose that $K \subset \mathbb{R}^{n}$ is closed, with non-empty interior $K^{\circ}$, and that $K$ has locally finite perimeter in $\mathbb{R}^{n}$. Suppose $K$ has a supporting hyperplane at each point $p$ of its reduced boundary $\partial_{\text {red }} K$. If $x \in \mathbb{R}^{n} \backslash K$, then $K$ has a supporting hyperplane $H=H(p, u)$, with $p \in \partial_{\text {red }} K$, such that $K \subset \overline{H_{-}(p, u)}$ and $x \in H_{+}(p, u)$.

Proof. If $x \in \mathbb{R}^{n} \backslash K$, then $U(x, r) \subset \mathbb{R}^{n} \backslash K$ for some $r>0$ since $\mathbb{R}^{n} \backslash K$ is open, and in particular dist $(x, K)>0$. Similarly, there exist $y \in \mathbb{R}^{n}$ and $R>0$ such that $U(y, R) \subset K^{\circ}$. For any $p \in \mathbb{R}^{n}$ with $p \neq x$ we let $\overrightarrow{x p}=\{(1-\lambda) x+\lambda p: \lambda \geq 0\}$ denote the ray through $p$ with initial point $x$, and we let

$$
\text { Cone }(x, U(y, R))=\left\{p \in \mathbb{R}^{n}: \overrightarrow{x p} \cap U(y, R) \neq \emptyset\right\}
$$

denote the cone with vertex $x$ through the ball $U(y, R)$. Let

$$
X=\text { Cone }(x, U(y, R)) \cap U(x,|x-y|) .
$$

$X$ is a bounded, convex portion of a cone, and $X \backslash\{x\}$ is open. Therefore, there exists a finite collection of open balls $V_{i}=U\left(c_{i}, r_{i}\right)$, for $i=1,2, \ldots, N$ (where $N \geq 3)$, such that $V_{1}=U(x, r), V_{N}=U(y, R), V_{i} \subset X$ for each $i=2, \ldots, N-1$, and $\mathcal{L}^{n}\left(V_{i} \cap V_{i+1}\right)>0$ for each $i=1,2, \ldots, N-1$. See Figure 1 .

Let $k=\min \left\{i: 1 \leq i \leq N, \mathcal{L}^{n}\left(K \cap V_{i}\right)>0\right\}$. We must have $k \geq 2$, since $\mathcal{L}^{n}\left(K \cap V_{1}\right)=0$, and we must have $k \leq N-1$ since $\mathcal{L}^{n}\left(K \cap V_{N}\right)=\alpha(n) R^{n}$ and $\mathcal{L}^{n}\left(V_{N-1} \cap V_{N}\right)>0$. By definition of $k, \mathcal{L}^{n}\left(K \cap V_{k-1}\right)=0$ and $\mathcal{L}^{n}\left(K \cap V_{k}\right)>0$. The former of these conditions implies $\mathcal{L}^{n}\left(\left(\mathbb{R}^{n} \backslash K\right) \cap V_{k-1}\right)=\alpha(n) r_{k-1}^{n}$, and so $\mathcal{L}^{n}\left(\left(\mathbb{R}^{n} \backslash K\right) \cap V_{k}\right)>0$, since $\mathcal{L}^{n}\left(V_{k-1} \cap V_{k}\right)>0$ by construction. Because $\mathcal{L}^{n}\left(K \cap V_{k}\right)>0$ and $\mathcal{L}^{n}\left(\left(\mathbb{R}^{n} \backslash K\right) \cap V_{k}\right)>0$, the relative isoperimetric inequality (7] 5.6.2, or 11 3.7.14) implies that $\mathcal{H}^{n-1}\left(\partial_{\text {red }} K \cap V_{k}\right)>0$, so that in particular there exists $p \in \partial_{\text {red }} K \cap V_{k}$. Since $2 \leq k \leq N-1$, we have $V_{k} \subset X \backslash\{x\}$, and so $p \in X \backslash\{x\}$. 


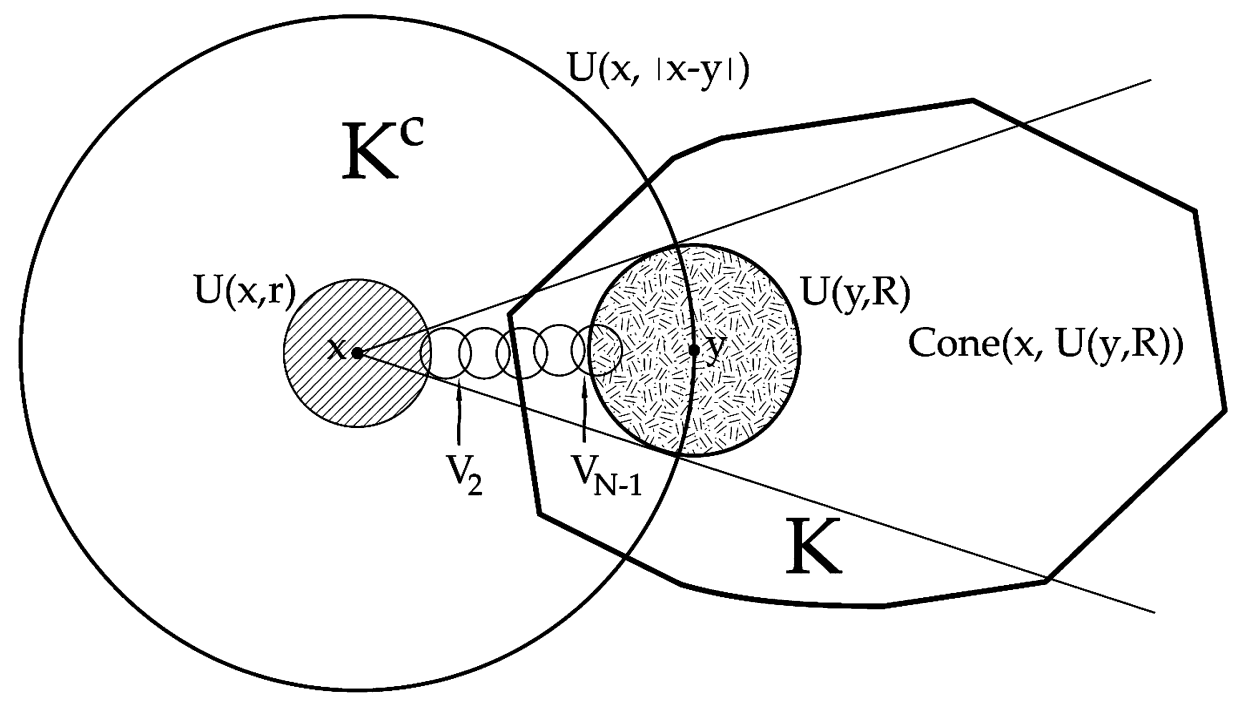

Figure 1

Let $H(p, u)$ be a supporting hyperplane to $K$ at $p$ such that $K \subset \overline{H_{-}(p, u)}$. By (3), there exists $z \in \overrightarrow{x p} \cap U(y, R)$, with $p \in \operatorname{relint} \overline{x z}$. We cannot have $x \in H_{-}(p, u)$, since then $p \in \overline{x z} \subset H_{-}(p, u)$, which contradicts $p \in H(p, u)$. We also cannot have $x \in H(p, u)$, for then we would have $\overrightarrow{x p} \in H(p, u)$ and therefore $z \in H(p, u)$, which contradicts $z \in K^{\circ}$.

If $K \subset \mathbb{R}^{n}$ is closed and has non-empty interior, and if $K$ has a supporting hyperplane at each point $p$ of its topological boundary $\partial K$, then $K$ is convex, and in fact it equals the intersection of all its supporting half-spaces $\overline{H_{-}(p, u)}$ where $p \in \partial K$ (cf. [12] Theorem 5.3, [14] Theorem 1.3.3 and Corollary 1.3.5). Theorem 2 will allow us to relax the hypotheses and at the same time strengthen the conclusion of this result. As before, we restrict our attention to the case of sets $K$ having locally finite perimeter, since otherwise $K$ is necessarily non-convex.

Theorem 3 (Representation theorem). Suppose that $K \subset \mathbb{R}^{n}$ is closed, with nonempty interior $K^{\circ}$, and that $K$ has locally finite perimeter in $\mathbb{R}^{n}$. Suppose $K$ has a supporting hyperplane at each point $p$ of its reduced boundary $\partial_{\text {red }} K$. Then $K=$ $\cap \overline{H_{-}(p, u)}$, where the intersection is taken over all supporting half-spaces $\overline{H_{-}(p, u)}$ where $p \in \partial_{\text {red }} K$. In particular, $K$ is convex.

Proof. If $K=\mathbb{R}^{n}$, then the theorem holds trivially, so suppose $K \neq \mathbb{R}^{n}$ and let $x \in \mathbb{R}^{n} \backslash K$. Then $K \subset \cap \overline{H_{-}(p, u)}$, where the intersection is taken over all supporting half-spaces $\overline{H_{-}(p, u)}$ where $p \in \partial_{\text {red }} K$. By Theorem 2 , $K$ has a supporting hyperplane $H=H(p, u)$, with $p \in \partial_{\text {red }} K$, such that $K \subset \overline{H_{-}(p, u)}$ and

$$
x \in H_{+}(p, u)=\mathbb{R}^{n} \backslash \overline{H_{-}(p, u)} \subset \mathbb{R}^{n} \backslash \cap \overline{H_{-}(p, u)} .
$$

Thus, $K=\cap \overline{H_{-}(p, u)}$, since $x$ was arbitrary. Since $K$ is the intersection of closed half-spaces, it must be convex. 
We now state our main theorem concerning convexity and reduced boundaries.

Theorem 4 (Convexity and reduced boundaries). Suppose $K \subset \mathbb{R}^{n}$ is closed, with non-empty interior $K^{\circ}$. Then the following conditions are equivalent:

a) $K$ is convex.

b) $K$ has locally finite perimeter in $\mathbb{R}^{n}$, and $K=\cap \overline{H_{-}(p, u)}$, where the intersection is taken over all supporting half-spaces $\overline{H_{-}(p, u)}$, where $p \in \partial_{\text {red }} K$.

c) $K$ has locally finite perimeter in $\mathbb{R}^{n}$, and $K$ has a supporting hyperplane at each point $p$ of its reduced boundary $\partial_{\text {red }} K$.

Proof. By Theorem [3, c) $\Rightarrow$ b) $\Rightarrow$ a). Now suppose $K$ is convex. We wish to show that $K$ has locally finite perimeter in $\mathbb{R}^{n}$. Suppose $\Omega$ is any open ball in $\mathbb{R}^{n}$. Without loss of generality, suppose $K \cap \bar{\Omega}$ has non-empty interior (or else $P(K, \Omega)=0)$. It follows that the topological boundary of the compact, convex set $K \cap \bar{\Omega}$ has finite area measure, since the surface area functional is continuous on the family of compact, convex subsets of $\mathbb{R}^{n}$ having non-empty interior ([12] 22.6), and so in fact the surface area of the topological boundary of $K \cap \bar{\Omega}$ is bounded above in terms of a function of the diameter of the set $K \cap \bar{\Omega}$. It then follows that $\mathcal{H}^{n-1}\left(\partial_{\text {red }} K \cap \Omega\right)<\infty$, as desired. Also, $K$ has a supporting hyperplane at each point $p$ of $\partial K$ (cf. [12] Theorem 5.4, [14] Theorem 1.3.2), hence at each point $p \in \partial_{\text {red }} K$, since $\partial_{\text {red }} K \subset \partial K$, so a) $\left.\Rightarrow \mathrm{c}\right)$.

Remark 5 (Nearest point projection). Theorem 4 relates the reduced boundary of $K$ to other properties of $K$ as well, since several conditions are equivalent to the convexity of a closed subset of $\mathbb{R}^{n}$ with non-empty interior. For instance, a), b), and c) are all equivalent to $K$ having a nearest point projection function $\pi_{K}: \mathbb{R}^{n} \rightarrow K$ with $\operatorname{Lip} \pi_{K}=1$ such that, corresponding to each $x \in \mathbb{R}^{n}, \pi_{K}(x)$ is the unique point $y$ in $K$ for which dist $(x, K)=|x-y|$.

\section{OTHER NOTIONS OF BOUNDARY}

If $X \subset \mathbb{R}^{n}$ is $\mathcal{L}^{n}$ measurable, we define the measure-theoretic interior, measuretheoretic exterior, and measure-theoretic boundary of $X$ as follows:

$$
\begin{aligned}
\operatorname{Int}(X) & =\left\{x \in \mathbb{R}^{n}: \Theta^{n}(X, x)=1\right\}, \\
\operatorname{Ext}(X) & =\left\{x \in \mathbb{R}^{n}: \Theta^{n}(X, x)=0\right\}, \\
\partial^{M} X & =\mathbb{R}^{n} \backslash(\operatorname{Int}(X) \cup \operatorname{Ext}(X)) .
\end{aligned}
$$

We define $X^{1 / 2}=\left\{x \in \mathbb{R}^{n}: \Theta^{n}(X, x)=1 / 2\right\}$. Also, $\partial_{F} X$ is the set of all points $p \in \mathbb{R}^{n}$ at which $X$ has a measure-theoretic exterior unit normal $u=n_{X}(p)$ in the sense of Federer (see 9] 4.5.5 and 4.5.6).

We can now extend the main results from the previous section to different types of boundaries, such as $\partial^{M} K, K^{1 / 2}$, and $\partial_{F} K$, each of which is widely used in geometric measure theory.

Theorem 6 (General separation theorem). Suppose that $K \subset \mathbb{R}^{n}$ is closed, with non-empty interior $K^{\circ}$, and that $K$ has locally finite perimeter in $\mathbb{R}^{n}$. Suppose $A$ is any subset of $\mathbb{R}^{n}$ for which $\partial_{\text {red }} K \subset_{n-1} A \subset \partial K$. Suppose $K$ has a supporting hyperplane at each point $p \in A$. If $x \in \mathbb{R}^{n} \backslash K$, then $K$ has a supporting hyperplane $H=H(p, u)$, with $p \in A$, such that $K \subset \overline{H_{-}(p, u)}$ and $x \in H_{+}(p, u)$. 
Proof. We define $U(x, r), U(y, R), X, V_{1}, V_{2}, \ldots, V_{N}$, and $k$ precisely as in the proof of Theorem 2] Because $\mathcal{L}^{n}\left(K \cap V_{k}\right)>0$ and $\mathcal{L}^{n}\left(\left(\mathbb{R}^{n} \backslash K\right) \cap V_{k}\right)>0$, the relative isoperimetric inequality ([7] 5.6.2 or [11] 3.7.14) and $\partial_{\text {red }} K \subset_{n-1} A$ imply that

$$
\mathcal{H}^{n-1}\left(A \cap V_{k}\right) \geq \mathcal{H}^{n-1}\left(\partial_{\text {red }} K \cap V_{k}\right)>0,
$$

so that in particular there exists $p \in A \cap V_{k}$. Since $2 \leq k \leq N-1$, we have $V_{k} \subset X \backslash\{x\}$, and so $p \in X \backslash\{x\}$. Let $H(p, u)$ be a supporting hyperplane to $K$ at $p$ such that $K \subset \overline{H_{-}(p, u)}$. By (3), there exists $z \in \overrightarrow{x p} \cap U(y, R)$, with $p \in \operatorname{relint} \overline{x z}$. We cannot have $x \in H_{-}(p, u)$, since then $p \in \overline{x z} \subset H_{-}(p, u)$, which contradicts $p \in H(p, u)$. We also cannot have $x \in H(p, u)$, for then we would have $\overrightarrow{x p} \in H(p, u)$ and therefore $z \in H(p, u)$, which contradicts $z \in K^{\circ}$.

Corollary 7 (Separation result for important special cases). Suppose that $K \subset \mathbb{R}^{n}$ is closed, with non-empty interior $K^{\circ}$, and that $K$ has locally finite perimeter in $\mathbb{R}^{n}$. Suppose $K$ has a supporting hyperplane at each point $p \in A$, where $A=K^{1 / 2}$, $A=\partial^{M} K, A=\partial_{F} K$, or $A=\partial K$. If $x \in \mathbb{R}^{n} \backslash K$, then $K$ has a supporting hyperplane $H=H(p, u)$, with $p \in A$, such that $K \subset \overline{H_{-}(p, u)}$ and $x \in H_{+}(p, u)$.

Proof. If $p \in \partial_{\text {red }} K$, then $K$ has a measure-theoretic exterior unit normal, $n_{K}(p)$, so that $\partial_{\text {red }} K \subset \partial_{F} K$. If $p \in \partial_{F} K$, then (9 4.5.5 and 4.5.6) imply that

$$
\Theta^{n}\left(H_{+}\left(p, n_{K}(p)\right) \cap K, p\right)=\Theta^{n}\left(H_{-}\left(p, n_{K}(p)\right) \backslash K, p\right)=0 .
$$

It follows that $\Theta^{n}(K, p)=\Theta^{n}\left(\mathbb{R}^{n} \backslash K, p\right)=1 / 2$, and thus $p \in K^{1 / 2}$, so that $\partial_{F} K \subset K^{1 / 2}$. Any $p \in K^{1 / 2}$ has density $1 / 2$, hence not 0 or 1 , so $K^{1 / 2} \subset$ $\partial^{M} K$. If $p \in \partial^{M} K$, then for each $r>0$ we have $\mathcal{L}^{n}(K \cap U(p, r))>0$ and $\mathcal{L}^{n}\left(\left(\mathbb{R}^{n} \backslash K\right) \cap U(p, r)\right)>0$, and in particular $K \cap U(p, r) \neq \emptyset$ and $\left(\mathbb{R}^{n} \backslash K\right) \cap$ $U(p, r) \neq \emptyset$, so $p \in \partial K$. Since $\partial_{\text {red }} K \subset \partial_{F} K \subset K^{1 / 2} \subset \partial^{M} K \subset \partial K$, we can now apply Theorem [6] with $A=K^{1 / 2}, A=\partial^{M} K, A=\partial_{F} K$, and $A=\partial K$ to complete the proof.

We can similarly extend Theorem 4 to any type of boundary which $\mathcal{H}^{n-1}$ almost contains the reduced boundary and which is a subset of the topological boundary.

Theorem 8 (Convexity and equivalent conditions). Suppose that $K \subset \mathbb{R}^{n}$ is closed, with non-empty interior $K^{\circ}$, and that $K$ has locally finite perimeter in $\mathbb{R}^{n}$. Suppose $A$ is any subset of $\mathbb{R}^{n}$ for which $\partial_{\text {red }} K \subset_{n-1} A \subset \partial K$. Then the following conditions are equivalent:

a) $K$ is convex.

b) $K=\cap \overline{H_{-}(p, u)}$, where the intersection is taken over all supporting halfspaces $\overline{H_{-}(p, u)}$, where $p \in A$.

c) $K$ has a supporting hyperplane at each point $p \in A$.

Proof. If $K=\mathbb{R}^{n}$, then $A=\emptyset$, so the theorem holds trivially. Suppose $K \neq \mathbb{R}^{n}$. If $K$ is convex, it has a supporting hyperplane at each point $p$ of $\partial K$, hence at each point $p \in A$, since $A \subset \partial K$, so a) $\Rightarrow$ c). Suppose c) holds, and let $x$ be an arbitrary point in $\mathbb{R}^{n} \backslash K$. Since $K$ is a subset of each of the $\overline{H_{-}(p, u)}$ 's, it follows that $K \subset \cap \overline{H_{-}(p, u)}$, where the intersection is taken over all supporting half-spaces $\overline{H_{-}(p, u)}$ where $p \in A$. By Theorem $6, K$ has a supporting hyperplane $H=H(p, u)$, with $p \in A$, such that $K \subset \overline{H_{-}(p, u)}$ and

$$
x \in H_{+}(p, u)=\mathbb{R}^{n} \backslash \overline{H_{-}(p, u)} \subset \mathbb{R}^{n} \backslash \cap \overline{H_{-}(p, u)} .
$$


Thus, $K=\cap \overline{H_{-}(p, u)}$, since $x$ was arbitrary, so c) $\Rightarrow$ b). Finally, b) $\Rightarrow$ a) since the convex sets are closed under intersections.

Corollary 9 (Convexity and equivalent conditions for important special cases). Suppose that $K \subset \mathbb{R}^{n}$ is closed, with non-empty interior $K^{\circ}$, and that $K$ has locally finite perimeter in $\mathbb{R}^{n}$. Suppose $A=K^{1 / 2}, A=\partial^{M} K, A=\partial_{F} K$, or $A=\partial K$. Then conditions $a$ ), $b$ ), and $c$ ) of Theorem 8 are equivalent.

Proof. The claim follows immediately from Theorem 8, since $\partial_{\text {red }} K \subset \partial_{F} K \subset$ $K^{1 / 2} \subset \partial^{M} K \subset \partial K$, as shown in the proof of Corollary 7

Remark 10. The comments in Remark 5 apply to Theorem 8 as well.

\section{ACKNOWLEDGMENTS}

The author would like to gratefully acknowledge useful suggestions made by the referee and by Tatiana Toro in preparing this paper for publication.

\section{REFERENCES}

[1] L. Ambrosio, N. Fusco, D. Pallara, Functions of bounded variation and free discontinuity problems, Oxford University Press, 2000. MR.1857292 (2003a:49002)

[2] D. G. Caraballo, Crystals and polycrystals in $\mathbb{R}^{n}$ : lower semicontinuity and existence, Journal of Geometric Analysis, 18, Number 1 (2008), 68-88. MR.2365668 (2009b:49032)

[3] D. G. Caraballo, Convexity, local simplicity, and reduced boundaries of sets, Journal of Convex Analysis, 18, no. 3 (2011), 823-832. MR2858096 (2012h:52004)

[4] D. G. Caraballo, On Tietze's convexity theorem and other local criteria for convexity, in preparation.

[5] E. De Giorgi, Su una teoria generale della misura $(r-1)$-dimensionale in uno spazio ad $r$ dimensioni, Ann. Mat. Pura Appl. (4), 36 (1954), 191-213, in Ennio De Giorgi: Selected Papers (L. Ambrosio, G. Dal Maso, M. Forti, M. Miranda, S. Spagnolo, eds.), Springer, 2006. MR0062214 (15:945d)

[6] E. De Giorgi, Nuovi teoremi relativi alle misure $(r-1)$-dimensionali in uno spazio ad $r$ dimensioni, Ricerche di Mat. 4 (1955), 95-113, in Ennio De Giorgi: Selected Papers (L. Ambrosio, G. Dal Maso, M. Forti, M. Miranda, S. Spagnolo, eds.), Springer, 2006. MR.0074499(17:596a)

[7] L. C. Evans, R. F. Gariepy, Measure theory and fine properties of functions, CRC Press, 1992. MR1158660 (93f:28001)

[8] H. Federer, The Gauss-Green Theorem, Transactions of the AMS, 58, No. 1 (1945), 44-76. $\operatorname{MR} 0013786(7: 199 \mathrm{~b})$

[9] H. Federer, Geometric measure theory, Springer-Verlag, 1969. MR0257325 (41:1976)

[10] Giusti, E., Minimal surfaces and functions of bounded variation, Birkhäuser, Boston, 1984. MR775682(87a:58041)

[11] S. G. Krantz, H. R. Parks, The geometry of domains in space, Birkhäuser, Boston, 1999. MR.1730695 (2000m:28005)

[12] S. R. Lay, Convex sets and their applications, Dover, 2007. MR.1170565 (93g:52001)

[13] P. Mattila, Geometry of sets and measures in Euclidean spaces: fractals and rectifiability, Cambridge University Press, 1995. MR 1333890 (96h:28006)

[14] R. Schneider, Convex bodies: the Brunn-Minkowski theory, Cambridge University Press, 1993. MR 1216521 (94d:52007)

[15] R. Webster, Convexity, Oxford University Press, 1994. MR.1443208 (98h:52001)

[16] M. L. Weitzman, An 'economics proof' of the supporting hyperplane theorem, Economics Letters, 68, Issue 1 (2000), 1-6. MR1765148(2001e:91048)

Department of Mathematics and Statistics, St. Mary's Hall, 3Rd floor, Georgetown University, Washington, DC 20057-1233 